\section{RECTAL SURGERY MADE SAFE FOR FOOLS.}

\section{BY EDMUND ANI)REWS, M.D., LL.D.,}

PROFESSOR OF CLINICAL SURGERY IN THE CHICAGO MEDICAL COLLEGE.

A few years ago I learned and published the secret method of treating piles employed by certain itinerants. The secret was sold from one quack to another at a high price, and consists generally in the hypodermic injection of various mixtures of carbolic acid. The hopes of many physicians that the method might prove a useful one, were greatly dampened by the discovery of about eighteen deaths out of some 3000 reported cases, and of very alarming symptoms in other instances; in short, it was the same fatal experience as that which previously put a stop to the injection of venous enlargements in other parts of the body.

Of late the Lake States are being treated to a new development; the little itinerant hypodermic syringe has budded and blossomed like Aaron's rod, and evolved little boxes of instruments and little books full of secret instructions, in short, little "systems" of rectal surgery, whereby, as one of the authors says, "operations which would otherwise be difficult can be accomplished with but little skill." The first style of boxes sells at a price varying from a hundred to a hundred and fifty dollars, according to the size of the purchaser's purse and credulity. They generally contain a hypodermic syringe and a rectal speculum, with a probe and a few other simple instruments, having some peculiarities of construction but no special excellence. An equally good set of instruments for the purpose would cost about fourteen dollars at retail, in the ordinary instrument stores.

It is curious to notice the obvious effort to combine a set of instruments in such a way that an ignorant purchaser may accomplish something with them but shall not be able to do much harm. Generally there are no cutting instruments whatever, and the only sharp weapons are the hypodermic syringe and the tenaculum. The chief formula for injection for hæmorrhoids is the following:

R. Acid carbol................... $3 \mathrm{j}$

Ol. olivæ....................... 3 v

Zinci chlorid ......................... gr. i m

Armed with his little box and book the wayfaring doctor, though a fool, may read and practice, and perchance make money, if the crop of piles is good, although the science of the colleges never glanced upon his calvarium.

Such an easy way to make money soon bred imitations. New boxes and new little books are in the market. Some contain several more instruments than those above mentioned, and yet are sold at half the price, being offered at about fifty dollars. There is, however, the same careful attention to the fact that the purchaser is expected to be such a fool that he cannot be trusted with edge tools, though some of the later boxes contain the little blunt-pointed bistoury of Sims' uterine set, with an edge about half an inch long, for a purpose presentiy to be mentioned. One set which I examined contained the following instruments :

I. A small rectal speculum.
2. A hypodermic syringe.

3. A tenaculum.

4. A small Sims' uterine knife, blunt pointed, and having an edge five-eighths of an inch long.

5. A Sims' flexible uterine probe, for examining fistula.

6. A blunt hook to pull down the "pockets" presently to be described.

7. A similar blunt hook with two minute barbs to hold the pocket when it is so shallow as to slip from the smooth hook.

8. A scarifying probe, made so as to scratch the interior walls of a fistula to the depth of about a sixteenth of an inch. By a blundering combination of Greek and Latin in the same word, forbidden by the usages of educated men, it is called a "fistulatome."

9. A flat scoop for clearing out the rectal pockets and pouches.

10. A ligature carrier, to facilitate ligating piles.

I I. A three ounce syringe.

12. A hard rubber tube for oiling the anus.

There are no scissors, probably because they could be dangerously used by ignorant purchasers.

- The following were some of the printed directions in the box:

"Radical. Cure of Fistula in Ano.-First trace fistula with flexible probe. Wash out the track with a 5 per cent. solution of 'hydrogen peroxide.' Then inject a 95 per cent. sol. of carbolic acid, plus equal quan. of a io per cent. sol. of muriate of cocaine. Draw about 10 to 15 minims in the syringe. Push the flexible needle to the depth of fistula, then inject slowly as you withdraw the needle. Within two hours inject oleum eucalyptus and glycerine, equal parts, and the operation is finished. Keep patient quiet for forty-eight hours."

"HeMORRHOIDS.-Hæmorrhoidal tumors should be injected with an eight grain sol. of muriate of cocaine, plus an equal volume of 'phenol sodique;' use of the injection from twenty minims to a drachm, according to size of tumor. It is seldom necessary to inject more than once or twice. This injection deposited in two and three drops, making the punctures one inch apart over the rectum, will seldom fail to cure prolapsus of the rectum. Should be repeated two or three times.

"During the treatment of hæmorrhoids or prolapsus, patient should take each night at bed-time a oneeighth grain pill of the solid extract leptandrin, and the parts should be kept anointed with 'ceratum taralinum,' once a day for a week after the operation. After operation on the rectum of a female, the uterus should be dilated, and after like operation on the male rectum, metallic sounds should be passed as large as the urethra will admit; and in all cases where there are fissures of the anus, the sphinctors should be forcibly dilated for ten seconds, while patient is under the influence of an anæsthetic.

"With our improved instruments designed expressly for treating the rectum, operations that would otherwise be difficult can be accomplished with but little skill. Our speculum is the only one that is self-retaining, thereby enabling the operator to use both hands, at the same time exposing every part of the 
rectum, and not causing the patient great pain by and that "nowhere, so far as" he is "aware, are over-distension of the sphinctors."

they well described or properly noticed," hence he

The "Phenol Sodique" is a French name for an article made and sold in Philadelphia. It is simply a solution of impure carbolic acid. The term "Ceratum Taralinum" is an ignorant man's effort to construct a Latin name without knowing the language. The article to which it is applied is a blackish, untidy looking unguentum picis liquida, or tar ointment, but probably not made according to the officinal directions. Druggists inform me that it is sold as a pro. prietary article under the name "taroid."

A recent addition to these "systems of rectal surgery" consists in exploring the anus for the little pockets in the mucous membrane which exist normally just above the external sphinctor, pulling them down with the blunt hook, and splitting them down with the Sims' knife. Another addition consists in cutting off the little projections, or caruncles, which are often found at the same part, under the prepos terous claim that they are very injurious to the patient. The operators call these organs "pockets and fringes."

The possibilities of deception in this field are unlimited, since the patient can neither see the "pockets and fringes" for himself, nor dispute their alleged pestiferous influence. This practice originated in a paper read before a Society of irregulars, entitled "Rectal Pockets and Fringes." That the paper may be properly estimated I will recall to memory here the structure of the lower rectum, as described by authors, both in anatomy and surgery: Morgagni described, what any dissector can see, a reticulated columnar structure of the mucous membrane just above the external sphinctor. These ridges, or "columns of Morgagni," resemble the columnce carnea of the inner walls of the heart, but are very much smaller. Occasionally a probe will pass behind one of the columns, as under a bridge, but generally they are merely adherent pilasters, or ridges, running longitudinally but somewhat branched. They are described not only by Morgagni, but by "Curling on theRectum," "Allen's Human Anatomy," "Kelsey on the Rectum," etc. Kelsey says, p. ro: "Between the lower ends of the columner recti (columns of Morgagni) little arches are stretched, forming pouches. They are more developed in old people, and may retain small pieces of freces, and give rise to suppuration and abscess."

Curling says, $\mu .6$, that between the lower ends of these columna recti " the mucous membrane is slight-! ly dilated, variously in different subjects, but in many to such an extent as to form small sacs, or pouches; and in the spaces between them there is a series of short projecting columnar processes about three. eighths of an inch in length."

The latter are analogous to the caruncula myrtiformes of the vagina. The writer of the paper seized on these "pockets and fringes," as he called the pouches and caruncles, and declared that "our current literature contains little or no mention of them." In spite of the fact that they are natural organs, and therefore must have a use, he asserts that "they are dent occurred, three quarters of an hour before I saw more prolific of mischief than you would believe," the patient. 